International Journal of Pure and Applied Mathematics

Volume 86 No. 1 2013, 83-94

ISSN: $1311-8080$ (printed version); ISSN: 1314-3395 (on-line version)

url: http://www.ijpam.eu

doi: http://dx.doi.org/10.12732/ijpam.v86i1.8

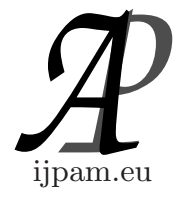

\title{
JACOBIAN-FREE ACCELERATING CONVERGENCE METHOD FOR NONLINEAR SYSTEM OF EQUATIONS
}

\author{
K.V. Narasimham \\ Department of Mathematics \\ Lendi Institute of Engineering and Technology \\ Vizianagaram, 535 005, A.P., INDIA
}

\begin{abstract}
In this paper, we present a Jacobian free accelerating convergence of the successive approximation method for solving nonlinear system of equations. The set of parameters is introduced for successive approximation method as consequence accelerating convergence is achieved. The convergence condition and rate of convergence are derived analytically. Some numerical illustrations are given and we compared these methods with Newton's method and successive approximation method.
\end{abstract}

AMS Subject Classification: $65 \mathrm{H} 10$

Key Words: non-linear equations, Newton-Raphson method, successive approximation method

\section{Introduction}

The real world problems when modeled mathematically yield complex dynamical systems. Their mathematical descriptions reduce to systems of algebraic, transcendental, functional and differential equations (may be linear or nonlinear).

It is well known that in recent years there has been a considerable interest in various higher order methods for solving nonlinear system of equations.

Received: March 15, 2013

(c) 2013 Academic Publications, Ltd. url: www.acadpubl.eu 
Narasimham., K.V achieved the Accelerating Convergence for Newton's Method with third order, successive approximation method for solving nonlinear scalar equations in [7] and [8].

We have considered the nonlinear system of equations of the following

$$
F(X)=0
$$

where $\left.F(X)=\left(F_{1} X\right), \ldots, F_{n}(X)\right)^{T}$ and $X=\left(x_{1}, \ldots, x_{n}\right)^{T}$

For the successive approximation method the system (1) can be written as

$$
x_{i}=f_{i}\left(x_{1}, \ldots, x_{n}\right), i=1,2 \ldots, n
$$

The system (2) converges if the following condition is satisfied [3]

$$
\sum_{j=1}^{n}\left|\frac{\partial \mathrm{f}_{\mathrm{i}}}{\partial x_{\mathrm{j}}}\right|<1, i=1,2 \ldots, n
$$

The Multi Variable Newton-Raphson Method [5]and[6]for the solution of (1) is given by

$$
X^{(k+1)}=X^{(k)}-\left[J^{(k)}\right]^{-1} F\left(X^{(k)}\right)
$$

where $J^{(k)}=\left(\frac{\partial F_{i}}{\partial x_{j}}\right)$ for each $i$ and $j$. The system (4) converges if $\left[J^{(k)}\right]^{-1}$ exists.

\section{Preliminary Results}

Definition 1. [1] Let $X^{*} \in \mathbb{R}^{m}, X_{n} \in \mathbb{R}^{m}, n=0,1 \ldots$ then the sequence $\left\{X_{n}\right\}$ is said to converge to $X^{*}$ if $\lim _{n \rightarrow \infty}\left\|X_{n}-X^{*}\right\|=0$. If, in addition there exists a constant $c=0$, an integer $n_{0}=0$, and $\rho=0$ such that for all $n>$ $n_{0},\left\|X_{n+1}-\mathrm{X}^{*}\right\| \leq c\left\|X_{n}-\mathrm{X}^{*}\right\|^{\rho}$, then $\left\{X_{n}\right\}$ is said to converge to $X^{*}$ with qorder at least $\rho$. If $\rho$ is 2 or 3 , the convergence is said to be q-quadratic or q-cubic respectively. When $E_{n}=X_{n}-X^{*}$ is the error in the $n^{\text {th }}$ iteration, the relation $E_{n+1}=c E_{n}^{\rho}+O\left(E_{n}^{\rho+1}\right)$ is called the error equation. By substituting $E_{n}=X_{n}-X^{*}$ for all $\mathrm{n}$ in any iteration method and simplifying, we obtain the error equation for that method. The value of $\rho$ thus obtained is called the order of this method.

Definition 2. [10] Let $X^{*}$ be a solution of the system $F(x)=0$ and suppose that $X_{n+1}, X_{n}$ and $X_{n-1}$ are three consecutive iteration closes to the root $X^{*}$, then the Computational Order of Convergence (COC) $\bar{\rho}$ can be approximated using the formula

$$
\bar{\rho}=\frac{\left.\ln \left[\| X_{n+1}-\mathrm{X}^{*}\right)\|/\| X_{n}-\mathrm{X}^{*} \|\right]}{\ln \left[\left\|X_{n}-\mathrm{X}^{*}\right\| /\left\|X_{n-1}-\mathrm{X}^{*}\right\|\right]} .
$$


Definition 3. [2] and [4] Let $X^{*}$ be a solution of the system $F(X)=0$ and suppose that $X_{n+1}, X_{n}$ and $X_{n-1}$ are three consecutive iteration closes to the root $X^{*}$, then the Approximated computational order of convergence (ACOC) $\widehat{\rho}$ can be approximated using the formula

$$
\widehat{\rho}=\frac{\ln \left[\left\|X_{n+1}-X_{n}\right\|\right] / \ln \left[\left\|X_{n}-X_{n-1}\right\|\right]}{\ln \left[\left\|X_{n}-X_{n-1}\right\|\right] / \ln \left[\left\|X_{n-1}-X_{n-2}\right\|\right.} .
$$

Stopping Criteria. We have to accept an approximate solution rather than the exact root, depending on the precision $(\epsilon)$ of the computer so, we use the following stopping criteria for computer programs:(i) $\left\|X_{n+1}-\mathrm{X}^{*}\right\|<\varepsilon$ (ii) $\left\|F\left(X_{n+1}\right)\right\|<\varepsilon$

\section{Jacobian-Free Accelerating Convergence Method for Nonlinear System of Equations}

Let $X^{*}=\left(x_{1}^{*}, \ldots, x_{n}^{*}\right)$ be a solution of the system (2), and in the region $R$ : $\left(x_{i}^{*}-h, x_{i}^{*}+h\right), i=1,2, \ldots, n$ such that

$$
\sum_{j=1}^{n}\left|\frac{\partial \mathrm{f}_{\mathrm{i}}}{\partial x_{\mathrm{j}}}\right|<\gamma<1, i=1,2, \ldots, n .
$$

Let us consider a set of non-negative parameters $\alpha_{i} \in\left(0,1 / \lambda_{i}\right]$, such that

$$
\lambda_{i}=\sum_{j=1, j \neq i}^{n}\left|\frac{\partial \mathrm{f}_{\mathrm{i}}}{\partial x_{\mathrm{j}}}\right|(\neq 0), i=1,2, \ldots, n .
$$

and

$$
x_{i}^{(k+1)}=\left(1-\alpha_{i}^{(k)}\right) x_{i}^{(k)}+\alpha_{i}^{(k)} x_{i}, i=1,2, \ldots, n .
$$

From (2) and (6), we have

$$
x_{i}^{(k+1)}=\left(1-\alpha_{i}^{(k)}\right) x_{i}^{(k)}+\alpha_{i}^{(k)} f_{i}\left(x_{1}^{(k)}, \ldots, x_{n}^{(k)}\right), i=1,2, \ldots, n .
$$

Let $\phi_{i}\left(x_{1}^{(k)}, \ldots, x_{n}^{(k)}\right)=\left(1-\alpha_{i}^{(k)}\right) x_{i}{ }^{(k)}+\alpha_{i}^{(k)} f_{i}\left(x_{1}^{(k)}, \ldots, x_{n}^{(k)}\right)$.

Now, $x_{i}^{(k+1)}-x_{i}^{*}=\phi_{i}\left(x_{1}^{(k)}, \ldots, x_{n}^{(k)}\right)-\phi_{i}\left(x_{1}^{*}, \ldots, x_{n}^{*}\right)$ and applying the law of mean for a function of $\mathrm{n}$ variables, we have

$$
x_{i}^{(k+1)}-x_{i}^{*}=\left(\frac{\partial \phi_{i}}{\partial x_{1}}\right)_{\xi^{k}}\left(x_{1}^{(k)}-x_{1}^{*}\right)+\cdots+\left(\frac{\partial \phi_{i}}{\partial x_{n}}\right)_{\xi^{k}}\left(x_{n}{ }^{(k)}-x_{n}^{*}\right),
$$


where $\xi^{k}=\left(\xi_{1}^{k}, \ldots, \xi_{n}^{k}\right), \xi_{i}^{k}$ lies between $x_{i}{ }^{(k)}$ and $x_{i}^{*}$, so that $\xi^{k}=\left(\xi_{1}^{k}, \ldots, \xi_{n}^{k}\right)$ is in $\mathbb{R}^{n}$. Then, representing the largest of the quantities, $\left|x_{i}^{(k)}-x_{i}^{*}\right|$, by $M^{(k)}$, we have

$$
\begin{aligned}
\left|x_{i}^{(k+1)}-x_{i}^{*}\right| & <\left|\frac{\partial \phi_{i}}{\partial x_{1}}+\cdots+\frac{\partial \phi_{i}}{\partial x_{n}}\right|\left|x_{i}^{(k)}-x_{i}^{*}\right| \\
& <\left|\left(1-\alpha_{i}\right)+\alpha_{i} \frac{\partial \mathrm{f}_{\mathrm{i}}}{\partial x_{i}}+\sum_{j=1, j \neq k}^{n} \alpha_{i} \frac{\partial \mathrm{f}_{\mathrm{i}}}{\partial x_{\mathrm{j}}}\right| M^{(k)} \\
& <\left\lceil\left|\left(1-\alpha_{i}\right)+\alpha_{i} \frac{\partial \mathrm{f}_{\mathrm{i}}}{\partial x_{i}}\right|+\sum_{j=1, j \neq k}^{n}\left|\alpha_{i} \frac{\partial \mathrm{f}_{\mathrm{i}}}{\partial x_{\mathrm{j}}}\right|\right]^{2} M^{(k-1)} \ldots \\
& <\left[\left|\left(1-\alpha_{i}\right)+\alpha_{i} \frac{\partial \mathrm{f}_{\mathrm{i}}}{\partial x_{i}}\right|+\sum_{j=1, j \neq k}^{n}\left|\alpha_{i} \frac{\partial \mathrm{f}_{\mathrm{i}}}{\partial x_{\mathrm{j}}}\right|\right]^{k} M^{(0)} .
\end{aligned}
$$

The convergence follows from these inequalities,

$$
\left|\left(1-\alpha_{i}\right)+\alpha_{i} \frac{\partial \mathrm{f}_{\mathrm{i}}}{\partial x_{i}}\right|+\sum_{j=1, j \neq k}^{n}\left|\alpha_{i} \frac{\partial \mathrm{f}_{\mathrm{i}}}{\partial x_{\mathrm{j}}}\right|<1, i=1,2, \ldots, n .
$$

For $\mathrm{i}=\mathrm{k}$, the condition (8) reduces to

$$
\left|1-\alpha_{k}+\alpha_{k} \frac{\partial \mathrm{f}_{\mathrm{k}}}{\partial x_{\mathrm{k}}}\right|+\alpha_{k} \sum_{j=1, j \neq k}^{n}\left|\frac{\partial \mathrm{f}_{\mathrm{k}}}{\partial \mathrm{x}_{\mathrm{j}}}\right|<1 .
$$

By rewriting (9), we get

$$
1-\alpha_{k} \sum_{j=1, j \neq k}^{n}\left|\frac{\partial \mathrm{f}_{\mathrm{k}}}{\partial x_{\mathrm{j}}}\right|>\left|1-\alpha_{k}+\alpha_{k} \frac{\partial \mathrm{f}_{\mathrm{k}}}{\partial x_{\mathrm{k}}}\right| .
$$

But

$$
1-\alpha_{k} \sum_{j=1, j \neq k}^{n}\left|\frac{\partial \mathrm{f}_{k}}{\partial x_{\mathrm{j}}}\right| \geq 0
$$

From (10) and (11), we get

$$
\left|1-\alpha_{k}+\alpha_{k} \frac{\partial \mathrm{f}_{\mathrm{k}}}{\partial x_{\mathrm{j}}}\right|=0
$$


By simplifying (12), we have

$$
\alpha_{k}=\left[1-\frac{\partial f_{k}}{\partial x_{k}}\right]^{-1}(\neq 0), k=1,2, \ldots, n .
$$

Theorem 4. Let us assume that the system (2) has a solution, then the Accelerating parameters $\alpha_{i}, i=1,2, \ldots, n$, are always non-negative and $\alpha_{i} \in\left(0,1 / \lambda_{i}\right]$, where

$$
\lambda_{i}=\sum_{j=1, j \neq i}^{n}\left|\frac{\partial \mathrm{f}_{\mathrm{i}}}{\partial x_{\mathrm{j}}}\right|(\neq 0) .
$$

Proof. From (2), we have $\left|\frac{\partial \mathrm{f}_{\mathrm{i}}}{\partial x_{\mathrm{i}}}\right|<1, i=1,2, \ldots, n$.

Case(i) Let $0<\frac{\partial \mathrm{f}_{\mathrm{i}}}{\partial x_{\mathrm{i}}}<1, i=1,2, \ldots, n$.

This gives that $\alpha_{i}=\left[1-\frac{\partial f_{i}}{\partial x_{i}}\right]^{-1}$ is positive and $\alpha_{i}>1, i=1,2, \ldots, n$. And $1-\frac{\partial \mathrm{f}_{\mathrm{i}}}{\partial x_{\mathrm{i}}} \geq 1-\left|\frac{\partial \mathrm{f}_{\mathrm{i}}}{\partial x_{\mathrm{i}}}\right|, i=1,2, \ldots, n$.

Since

$$
\sum_{j=1}^{n}\left|\frac{\partial \mathrm{f}_{\mathrm{i}}}{\partial x_{\mathrm{j}}}\right|<1, i=1,2, \ldots, n,
$$

then

$$
\sum_{j=1, j \neq i}^{n}\left|\frac{\partial \mathrm{f}_{\mathrm{i}}}{\partial x_{\mathrm{j}}}\right|+\left|\frac{\partial \mathrm{f}_{\mathrm{i}}}{\partial x_{\mathrm{i}}}\right|<1
$$

This implies that,

$$
\sum_{j=1, j \neq i}^{n}\left|\frac{\partial \mathrm{f}_{\mathrm{i}}}{\partial x_{\mathrm{j}}}\right|<1-\left|\frac{\partial \mathrm{f}_{\mathrm{i}}}{\partial x_{\mathrm{i}}}\right|
$$

Hence,

$$
\frac{1}{1-\frac{\partial \mathrm{f}_{\mathrm{i}}}{\partial x_{\mathrm{i}}}}<\left[\sum_{j=1, j \neq i}^{n}\left|\frac{\partial \mathrm{f}_{\mathrm{i}}}{\partial x_{\mathrm{j}}}\right|^{-1}, i=1,2, \ldots, n .\right.
$$

This gives $\alpha_{i}<1 / \lambda_{i}, \quad i=1,2, \ldots, n$.

Case (ii) Let $-1<\frac{\partial \mathrm{f}_{\mathrm{i}}}{\partial x_{\mathrm{i}}}<0, i=1,2, \ldots, n$.

Clearly, $\alpha_{i}=\left[1-\frac{\partial f_{i}}{\partial x_{i}}\right]^{-1}$ is positive and $\frac{1}{2}<\alpha_{i}, i=1,2, \ldots, n$.

Case (iii) Let $\frac{\partial \mathrm{f}_{\mathrm{i}}}{\partial x_{\mathrm{i}}}=0$. (i.e.,) $\alpha_{i}=1$.

This implies that no acceleration is achieved. 


\subsection{Convergence Condition}

Theorem 5. The Jacobian-Free Accelerating Convergence of successive approximation method will always converge for

$$
\alpha_{i}=\left[1-\frac{\partial f_{i}}{\partial x_{i}}\right]^{-1}
$$

for each $i$, if the system(2) is convergent.

Proof. Let us assume the system (2) is convergent.

Let

$$
\varphi_{i}(X)=\left(1-\alpha_{i}^{(k)}\right) x_{i}^{(k)}+\alpha_{i}^{(k)} f_{i}\left(x_{1}^{(k+1)}, \ldots, x_{i-1}^{(k+1)}, x_{i}^{(k)}, \ldots, x_{n}^{(k)}\right)
$$

for each $i=1,2, \ldots, n$.

Then the system (7) takes the form

$$
x_{i}^{(k+1)}=\varphi_{i}\left(x_{1}^{(k+1)}, \ldots, x_{i-1}^{(k+1)}, x_{i}^{(k)}, \ldots, x_{n}^{(k)}\right), i=1,2, \ldots, n .
$$

Now,

$$
\sum_{j=1}^{n}\left|\frac{\partial \varphi_{\mathrm{i}}}{\partial x_{\mathrm{j}}}\right|=\left|1-\alpha_{i}+\alpha_{i} \frac{\partial \mathrm{f}_{i}}{\partial x_{\mathrm{i}}}\right|+\sum_{j=1, j \neq i}^{n}\left|\alpha_{i} \frac{\partial \mathrm{f}_{\mathrm{i}}}{\partial \mathrm{x}_{\mathrm{j}}}\right|, i=1,2, \ldots, n .
$$

Hence,

$$
\sum_{j=1}^{n}\left|\frac{\partial \varphi_{\mathrm{i}}}{\partial x_{\mathrm{j}}}\right|=\sum_{j=1, j \neq i}^{n}\left|\alpha_{i} \frac{\partial \mathrm{f}_{\mathrm{i}}}{\partial \mathrm{x}_{\mathrm{j}}}\right|<1
$$

\subsection{Rate of Convergence}

Theorem 6. Let $f_{i}: D \subseteq R^{n} \rightarrow R$ be continuously differentiable functions such that

$$
\sum_{j=1}^{n}\left|\frac{\partial \mathrm{f}_{\mathrm{i}}}{\partial x_{\mathrm{j}}}\right|<\gamma<\frac{1}{3}<1
$$

for each $i=1,2, \ldots, n$, then the Accelerated Convergence of successive approximation method defined by (3.3), $\alpha_{i}^{(k)}=\left[1-\frac{\partial f_{i}^{*}}{\partial x_{i}}\right]^{-1}$ and $f_{i}^{*}=f_{i}\left(x_{1}^{*}, \ldots, x_{n}^{*}\right)$, $x_{i}^{*}=f_{i}\left(x_{1}{ }^{(k)}, \ldots, x_{n}^{(k)}\right)$ for $k \geq 0$ converges to $X^{*}=\left(x_{1}^{*}, \ldots, x_{n}^{*}\right)^{T}$. 
Proof. Since $f_{i}: D \subseteq R^{n} \rightarrow R$, the sequence $\left\{X^{(k)}\right\}_{k=1}^{\infty}$, where $X=$ $\left(x_{1}, \ldots, x_{n}\right)^{T}$, is defined for all $k>0$. Now,

$$
\sum_{i=1}^{n}\left|x_{i}^{(k+1)}-x_{i}^{*}\right|=\sum_{i=1}^{n}\left(1-\alpha_{i}^{(k)}\right) x_{i}^{(k)}+\alpha_{i}^{(k)} f_{i}\left(X^{(k)}\right)-x_{i}^{*},
$$

where $f_{i}\left(X^{(k)}\right)=f_{i}\left(x_{1}^{(k+1)}, \ldots, x_{i-1}^{(k+1)}, x_{i}^{(k)}, \ldots, x_{n}^{(k)}\right)$.

This implies

$$
\begin{aligned}
\sum_{i=1}^{n}\left|x_{i}^{(k+1)}-x_{i}^{*}\right| & =\sum_{i=1}^{n} \alpha_{i}^{(k)}\left(f_{i}\left(X^{(k)}\right)-x_{i}^{*}\right)+\left(1-\alpha_{i}^{(k)}\right)\left(x_{i}^{(k)}-x_{i}^{*}\right) \\
& =\sum_{i=1}^{n}\left[\alpha_{i}^{(k)} f_{i}\left(X^{(k)}\right)-x_{i}^{*}+1-\alpha_{i}^{(k)} x_{i}^{(k)}-x_{i}^{*}\right] \\
& =\sum_{i=1}^{n}\left[\alpha_{i}^{(k)} f_{i}\left(X^{(k)}\right)-f_{i}\left(X^{*}\right)+1-\alpha_{i}^{(k)} x_{i}^{(k)}-x_{i}^{*}\right] \\
& =\sum_{i=1}^{n} \alpha^{(k)} x^{(k)}-x^{*} \sum_{j=1}^{n}\left|\frac{\partial \mathrm{f}_{\mathrm{i}}^{*}}{\partial x_{\mathrm{j}}}\right|+1-\alpha^{(k)} x^{(k)}-x^{*}
\end{aligned}
$$

This implies that

$$
\begin{aligned}
\sum_{i=1}^{n}\left|x_{i}^{(k+1)}-x_{i}^{*}\right| & <\sum_{i=1}^{n}\left[\gamma \alpha_{i}^{(k)} x_{i}^{(k)}-x_{i}^{*}+1-\alpha_{i}^{(k)} x_{i}^{(k)}-x_{i}^{*}\right] \\
& <\sum_{i=1}^{n} x_{i}^{(k)}-x_{i}^{*}\left(\gamma \alpha_{i}^{(k)}+1-\alpha_{i}^{(k)}\right) \\
& <\sum_{i=1}^{n} x_{i}^{(k)}-x_{i}^{*}\left[\frac{\gamma+\left|\frac{\partial \mathrm{f}_{i}^{*}}{\partial x_{x}}\right|}{\left|1-\frac{\partial \mathrm{f}_{i}^{*}}{\partial x_{x}}\right|} \mid\right.
\end{aligned}
$$

Thus,

$$
\sum_{i=1}^{n}\left|x_{i}^{(k+1)}-x_{i}{ }^{*}\right|<\sum_{i=1}^{n} x_{i}^{(k)}-x_{i}^{*}\left[\frac{2 \gamma}{1-\gamma}\right]
$$

Therefore,

$$
\sum_{i=1}^{n}\left|x_{i}^{(k)}-x_{i}^{*}\right|<\left[\frac{2 \gamma}{1-\gamma}\right] \sum_{i=1}^{n} x_{i}^{(k-1)}-x_{i}^{*}
$$




$$
\begin{aligned}
& <\left[\frac{2 \gamma}{1-\gamma}\right]^{2} \sum_{i=1}^{n}\left|x_{i}^{(k-2)}-x_{i}^{*}\right| \\
& <\left[\frac{2 \gamma}{1-\gamma}\right]^{3} \sum_{i=1}^{n}\left|x_{i}^{(k-3)}-x_{i}^{*}\right| \\
& \ldots \\
& <\left[\frac{2 \gamma}{1-\gamma}\right]^{k} \sum_{i=1}^{n}\left|x_{i}^{(0)}-x_{i}^{*}\right| .
\end{aligned}
$$

Since $0<\gamma<\frac{1}{3}<1$, we have $\lim _{k \rightarrow \infty}\left[\frac{2 \gamma}{1-\gamma}\right]^{k}\left|x^{(0)}-x^{*}\right|=0$.

Hence $\left\{X^{(k)}\right\}_{k=1}^{\infty}$ converges to $X^{*}$.

\section{Algorithm 1.}

The Jacobian-Free Accelerating convergence method is

$$
x_{i}^{(k+1)}=\left(1-\alpha_{i}^{(k)}\right) x_{i}^{(k)}+\alpha_{i}^{(k)} f_{i}\left(x_{1}^{(k+1)}, \ldots, x_{i-1}^{(k+1)}, x_{i}^{(k)}, \ldots, x_{n}^{(k)}\right),
$$

where $\alpha_{i}=\left[1-\frac{\partial f_{i}^{*}}{\partial x_{i}}\right]^{-1}$ and $f_{i}^{*}=f_{i}\left(x_{1}^{*}, \ldots, x_{n}^{*}\right), x_{i}^{*}=f_{i}\left(x_{1}, \ldots, x_{n}\right)$.

\section{Numerical Problems}

In this Section, we employ the new method, The Jacobian-Free Accelerating Convergence Method (JFACM) to solve nonlinear systems of equations and compare it with Newton-Raphson Method (NM), and successive approximation methods (SAM). All Numerical experiments were done in MATLAB with 15 digits precision.

Table 1 gives the comparison of the number of iterations (IT), functional evaluations (NFE), Computational Order of Convergence (COC), and Approximated Computational Order of Convergence (ACOC) of Test Problem 1.The solution and initial guess of Test Problem 2 are listed in Table 2.The computational results show that the new methods require less IT and NFE than NM and SAM as far as the numerical results are concerned. Therefore, the new methods are of practical interest.

Test Problem 1. We consider the non-linear system of three equations in three unknowns[11],

$$
F_{1}\left(x_{1}, x_{2}, x_{3}\right)=3 x_{1}-\operatorname{Cos}\left(x_{2} x_{3}\right)-0.5=0,
$$




\begin{tabular}{|c|c|c|c|}
\hline \multicolumn{4}{|c|}{ Test Problem-1 } \\
\hline Parameter & Method & $X_{0}=(0,0,0)$ & $X_{0}=(1,1,0)$ \\
\hline IT & JFACM & 3 & 3 \\
& NM & $\infty$ & 10 \\
& SAM & 20 & 20 \\
\hline NFE & JFACM & 18 & 18 \\
& NM & $\infty$ & 120 \\
& SAM & 60 & 48 \\
\hline COC & JFACM & 1.5570 & 1.5570 \\
& NM & $\infty$ & 1.1154 \\
& SAM & 0.9884 & 0.9881 \\
\hline ACOC & JFACM & 0.5818 & 0.5818 \\
& NM & $\infty$ & 1.0136 \\
& SAM & 1.0111 & 0.9893 \\
\hline
\end{tabular}

Table 1

$$
\begin{aligned}
& F_{2}\left(x_{1}, x_{2}, x_{3}\right)=x_{1}^{2}-625 x_{2}^{2}=0, \\
& F_{3}\left(x_{1}, x_{2}, x_{3}\right)=e^{-x_{1} x_{2}}+20 x_{3}+9=0 .
\end{aligned}
$$

The solution of Test Problem 1 is

$$
\begin{aligned}
& x_{1}=0.499983367726846, \\
& x_{2}=0.019999334709074 \\
& x_{3}=-0.499502524620480 .
\end{aligned}
$$

Test Problem 2. Consider the integral equation [9]

$$
u(t)=1+\frac{1}{4} t u(t) \int_{0}^{1} \frac{u(s)}{s+t} d s
$$

This equation is a special case of the H-equations arising in the study of radiative transfer. We approximate this integral equation applying the trapezoidal rule at the $n+1$ equidistant nodes $i h$, where $h=1 / n$. Making use of $u(0)=1$, we obtain the nonlinear systems of equations.

$$
F_{i}(X)=x_{i}\left(c-\frac{h}{4} \sum_{j=1}^{n} w_{i j} x_{j}\right)^{-1, i=1,2, \ldots, n}
$$



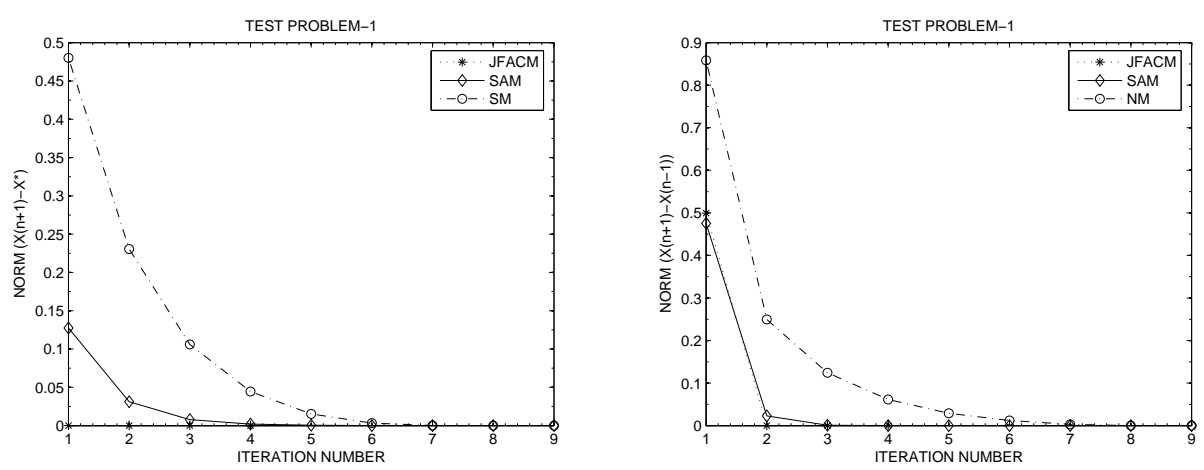

Figure 1: (a) \& (b)
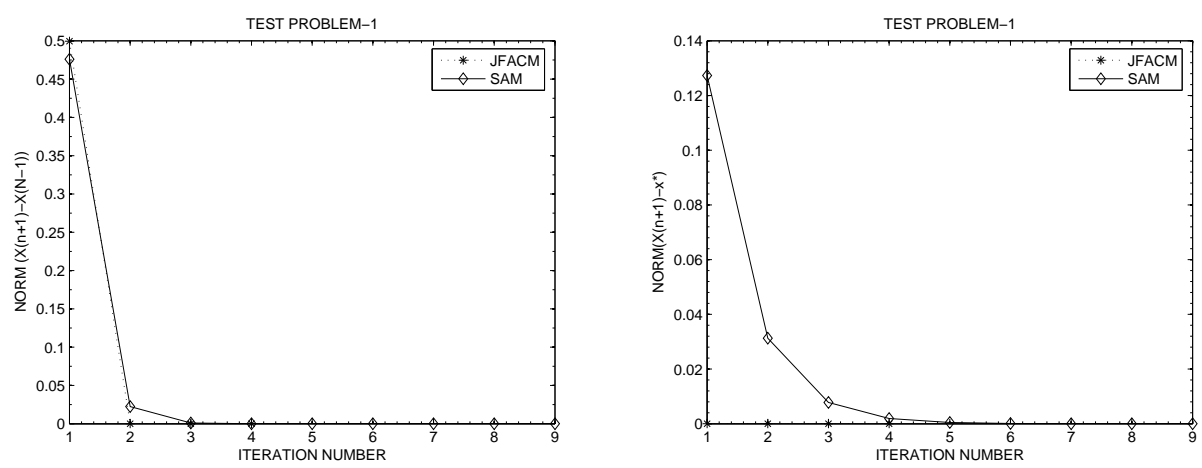

Figure 2: (a) \& (b)

with $c=\left(1-\frac{h}{8}\right), w_{i n}=\frac{i}{2(i+n)}$ and $w_{i j}=\frac{i}{i+j}$ for $j=1,2, \ldots, n-1$. Herein $x_{i}$ is an approximation of $u(i h)$. The above system (15) can be written as,

$$
f_{i}=x_{i}=\frac{\left[1+\frac{h}{4} x_{i} \sum_{j=1}^{n} w_{i j} x_{j}\right]}{c}, i=1,2, \ldots, n .
$$

The calculation was done on computer using MATLAB program of 15 deci-

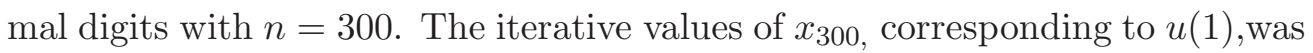
tabulated in Table 2

Remark. JFACM requires $n$ number of partial derivatives where as Classical Newton's Method (CNM) required $n^{2}$ number of partial derivatives for solving nonlinear systems of equations with $n$ variables, $n$ equations.

In Test Problem 2, 300 partial derivatives are used in JFACM and 90,000 partial derivatives are required for CNM. So, CNM is quite difficult to im- 


\begin{tabular}{|c|c|c|}
\hline & JFACM & SAM \\
\hline Initial Guess & $(1,1, \ldots, 1)$ & $(1,1, \ldots, 1)$ \\
\hline Solution $x_{300}$ & 1.251259561665 & 1.251259561665 \\
\hline IT & 9 & 19 \\
\hline
\end{tabular}

Table 2

plement for Test Problem 2 whereas JFACM is much suitable for solving the integral equation (14). Further we note that the inverse of the Jacobian matrix of order $300 \times 300$ is required for implementation of CNM but inverse matrix is not required for JFACM.

\section{Conclusion}

By computational analysis of Test Problem and results in Section 3, we observed that the introduction of acceleration parameters $\alpha_{i}$ forced the Acceleration of convergence of SAM for solving nonlinear systems of equations. Further, JFACM for Test Problem 1 is also applicable when classical NM fails or divergence cases, which is shown in Table 1. The introduction of appropriate relaxation parameters forced the convergence for the divergence causes of Test Problem 1. We conclude that the acceleration parameters $\alpha_{i}$ played a vital role in the Accelerating Convergence of SAM. These computational analyses show that the new Algorithm is more efficient and it performs better than classical Newton's method and some other methods.

\section{References}

[1] Jr.J.E. Dennis, B. Schnabel Robert, Numerical Methods for Unconstrained Optimization and Nonlinear Equations, SIAM, Philadelphia (1996).

[2] J.A. Ezquerro, M. Grau-Sanchez, A. Grau, M.A. Hernandez, M. Noguera, $\mathrm{N}$. Romero, On iterative methods with accelerated convergence for solving systems of nonlinear equations, J.Optim. Theory Appl., 151 (2011), 163174, doi: 10.1007/s10957-011-9870-y.

[3] L.R. Ford, The solution of equation by the method of successive approximation, Am.Math.Mon., 32 (1925), 272-287, doi: 10.2307/2299139. 
[4] M. Grau-Sanchez, M. Noguera, J.M. Gutierrez, On some computational orders of convergence, Appl.Math.Lett., 23 (2010), 472-478, doi: 10.1016/j.aml.2009.12.006.

[5] John Verbeke, Ronald Cools, The Newton-Raphson method, Int. J. Math. Edu. Sci. Technol., 26, No. 2 (1995), 177-193, doi: 10.1080/0020739950260202.

[6] Kendall E. Atkinson, An Introduction to Numerical Analysis, Wiley John Wiley \& Sons, Newyork (1989).

[7] K.V. Narasimham, Accelerating convergence of successive approximation method for nonlinear equations, Int. J. Comput. Appl. Math., 6 (2011), 149-160.

[8] K.V. Narasimham, Accelerating convergence of Newton's method with third order for nonlinear equations, Int. J. Numer. M. and Appl., 2 (2009), 59-65.

[9] J.M. Ortega, W.C. Rheinboldt, Iterative Solution of Nonlinear Equations in Several Variables, SIAM, Philadelphia (2000).

[10] S. Weerakoon, T.G.I. Frenando, A variant of Newton's method with accelerated third-order convergence, Appl.Math.Lett., 13 (2000), 87-93, doi: 10.1016/S0893-9659(00)00100-2.

[11] William W. Hager, Applied Numerical Linear Algebra, Prentice-Hall, Engle wood cliffs, NJ (1988). 